\title{
ARTICLE \\ Parallel droplet microfluidics for high throughput cell encapsulation and synthetic microgel generation
}

\author{
Devon M. Headen ${ }^{1,2}$, José R. García ${ }^{1,2}$ and Andrés J. García ${ }^{1,2}$
}

Cells can be microencapsulated in synthetic hydrogel microspheres (microgels) using droplet microfluidics, but microfluidic devices with a single droplet generating geometry have limited throughput, especially as microgel diameter decreases. Here we demonstrate microencapsulation of human mesenchymal stem cells (hMSCs) in small ( $<100 \mu$ m diameter) microgels utilizing parallel droplet generators on a two-layer elastomer device, which has $600 \%$ increased throughput vs. single-nozzle devices. Distribution of microgel diameters were compared between products of parallel vs. single-nozzle configurations for two square nozzle widths, 35 and $100 \mu \mathrm{m}$. Microgels produced on parallel nozzles were equivalent to those produced on single nozzles, with substantially the same polydispersity. Microencapsulation of hMSCs was compared for parallel nozzle devices of each width. Thirty five micrometer wide nozzle devices could be operated at twice the cell concentration of $100 \mu \mathrm{m}$ wide nozzle devices but produced more empty microgels than predicted by a Poisson distribution. Hundred micrometer wide nozzle devices produced microgels as predicted by a Poisson distribution. Polydispersity of microgels did not increase with the addition of cells for either nozzle width. hMSCs encapsulated on $35 \mu \mathrm{m}$ wide nozzle devices had reduced viability ( 70\%) and a corresponding decrease in vascular endothelial growth factor (VEGF) secretion compared to hMSCs cultured on tissue culture (TC) plastic. Encapsulating hMSCs using $100 \mu \mathrm{m}$ wide nozzle devices mitigated loss of viability and function, as measured by VEGF secretion.

Keywords: droplets; microencapsulation; microgel; multilayer; parallel

Microsystems \& Nanoengineering (2018) 4, 17076; doi:10.1038/micronano.2017.76; Published online: 15 January 2018

\section{INTRODUCTION}

Delivery of encapsulated proteins and cells in hydrogel microspheres, or microgels, has emerged as a promising therapeutic strategy for the treatment of various diseases ${ }^{1}$. Synthetic polymers provide tuneable release rates of encapsulated proteins that can be tailored based on diffusion through the hydrogel network or hydrolytic/enzymatic degradation of microgels ${ }^{2-4}$. These injectable microgels can be implanted in a minimally invasive manner for extended local delivery of therapeutic proteins, such as growth factors and cytokines. In addition, microgel delivery of proteins could allow for 'mosaic' injections, in which multiple microgel populations with individually validated release profiles are combined to provide more complex release profiles or multiprotein delivery.

Encapsulation of cells in microgels provides several key benefits in transplantation settings. By controlling mesh size of microgels, immune infiltration of transplanted cells can be mitigated while transport of oxygen and cellular waste products is maintained. Because potential sources of therapeutic cells are often allogeneic or xenogeneic, immunoisolation has been extensively investigated for reducing systemic immunosuppression requirements after transplantation 5,6 . Microgels can also function to immobilize cells at a desired transplant site by entrapping them in gels, an important consideration since many cell therapies currently rely on systemic administration of cells ${ }^{7}$. In addition to isolating transplanted cells from the host, synthetic microgels permit engineering of encapsulated cell microenvironment, including matrix stiffness and adhesive ligand interactions, which influence differentiation and secretory function of encapsulated stem cells $^{8,9}$. Bulk hydrogel constructs can provide these benefits as well, but the microgel form factor enables applications for which bulk gels are not well suited, such as injection. In addition, thick polymer coatings are a barrier to nutrients and oxygen, so hypoxic death is frequently observed in bulk gels, and even in large microgels, especially when high cell concentrations are used ${ }^{10}$. Therefore, microgel size should be minimized in order to maximize transport of oxygen to encapsulated cells and to enable novel, minimally invasive transplantation schemes. Encapsulation of cells in microgels with diameters $<100 \mu \mathrm{m}$ enables immobilization of cells in small blood vessels, including arterioles and larger capillaries. Alternatively, larger microgels may occlude larger vessels, restricting blood flow, and unencapsulated cells are difficult to localize ${ }^{7}$.

Alginate has been extensively explored for cell microencapsulation because rapid crosslinking allows for facile gelation in cationic aqueous solutions ${ }^{11}$. However, only limited control of cellular microenvironment is possible with alginate. We have previously developed a microfluidic droplet strategy for generating sizecontrolled crosslinked synthetic microgels based on 4-arm polyethylene glycol (PEG-4MAL) macromers, which are terminated with maleimides ${ }^{12}$. Maleimides efficiently react with free thiols to form covalent bonds, allowing any cysteine containing peptide or thiolated ligand to be tethered to microgels. This versatile chemistry enables local and sustained presentation of bioactive

\footnotetext{
${ }^{1}$ Woodruff School of Mechanical Engineering, Georgia Institute of Technology, Atlanta, GA 30332, USA and ${ }^{2}$ Petit Institute for Bioengineering and Biosciences, Georgia Institute of Technology, Atlanta, GA 30332, USA

Correspondence: Andrés J. García (andres.garcia@me.gatech.edu)

Received: 28 May 2017; revised: 23 August 2017; accepted: 29 August 2017
} 
molecules in microgels, as well as enzyme-mediated active release of therapeutic proteins when microgels are crosslinked with protease-sensitive crosslinks ${ }^{2}$. Crosslinking PEG-4MAL macromers can be accomplished with dithiol molecules and does not require free radical initiators, which are detrimental to encapsulated cell health ${ }^{13}$. Similar microfluidic schemes for producing microgels ${ }^{14-16}$ and encapsulating cells ${ }^{17-19}$ have recently been reported for widely varying applications, including wound healing, stem cell culture and fundamental studies of cell biology. The versatility of microfluidic cell encapsulation extends to other polymers, including Matrigel $^{20}$, agarose ${ }^{21}$ and even multilayer core-shell composites such as collagen cores with alginate shells ${ }^{22}$.

Minimization of microgel diameter maximizes transport of oxygen and nutrients to encapsulated cells, enables applications for which larger microgels are not suited (such as injection) and potentially reduces transplant graft volume. Microfluidics provide precise control of size and polydispersity of droplets ${ }^{23}$ and can be used to make very small cell laden microgels, including those with membranes as thin as $6 \mu \mathrm{m}^{24}$. Unfortunately, volumetric throughput of macromer on flow focusing nozzles decreases with the cube of droplet diameter, so volumetric throughput can be severely limited when very small microgels are being produced. Furthermore, cells suspended at high concentrations ( $>10^{6}$ cells per $\mathrm{mL}$ ) tend to aggregate, which can lead to incomplete cell encapsulation as well as increased microgel diameters. Therefore, low concentration cell suspensions $\left(10^{4}-10^{5}\right.$ cells per $\left.\mathrm{mL}\right)$ are typically used, often resulting in a high percentage of empty microgels according to the Poisson distribution ${ }^{25}$, which is undesirable for in vivo efficacy and limits applications of encapsulation. Microgels containing cells can be purified by sorting or can be selectively crosslinked ${ }^{7,24,26,27}$, but encapsulating clinically relevant numbers of cells in these very small microgels is highly challenging, since several tens of millions of cells may be required for therapeutic efficacy ${ }^{28,29}$. Long processing times associated with encapsulation or sorting are likely to decrease cell viability, so faster cell microencapsulation rates are desirable not just for convenience, but also to maintain cell potency.

To maximize cell encapsulation rate, both the concentration of cells in suspension and the volumetric throughput of macromer in microfluidic devices must be maximized. One way to increase flow rate of macromer in a microfluidic droplet generating device is to utilize a parallel flow focusing nozzle configuration. However, coupled inlet and outlet flow paths present significant challenges to maintaining size control and low polydispersity of droplet diameter, due to propagation of pressure fluctuations between nozzles. Nonetheless, uniform droplet production has been shown on parallel droplet generators with a variety of configurations ${ }^{30-34}$. Parallel microfluidics have been used to produce microgels through light-initiated free-radical polymerization on single layer microfluidics, and the nature of crosstalk between parallel droplet generators has been studied in this context ${ }^{35}$. Furthermore, in an impressive display of microfluidic control, even uniform double emulsion polymerosomes were formed on parallel geometries ${ }^{36,37}$, and robust, massively parallel droplet production has been demonstrated ${ }^{38}$. However, suspensions containing high density cell suspensions complicate fluid behavior and may cause non-uniform distribution of fluid to focusing nozzles. Likely because of these hurdles, parallel droplet generators have not been utilized to date for suspension of cells in droplets, much less their encapsulation in polymer microspheres.

Here we report a process for the high-throughput encapsulation of cells in sub-100 $\mu$ m diameter microgels, which utilizes a novel two-layer microfluidic device with six parallel flow focusing nozzles. Devices with two different nozzle widths, 35 and $100 \mu \mathrm{m}$, were developed, each with benefits and disadvantages. Thirty five micrometer wide nozzle devices produce smaller microgels and can break up droplets from precursors with twice the cell concentration as $100 \mu \mathrm{m}$ wide nozzle devices. However, encapsulation on $35 \mu \mathrm{m}$ wide nozzle devices reduces viability of human mesenchymal stem cells (hMSCs), and reduces their ability to secrete vascular endothelial growth factor (VEGF) in proportion to this loss of viability. On the other hand, following encapsulation on $100 \mu \mathrm{m}$ wide nozzle devices, hMSCs have $>90 \%$ viability and only minimal reduction of VEGF secretion vs. hMSCs cultured on tissue culture (TC) plastic. Although only six parallel channels are utilized in order to maintain robust operation, this parallelization of $100 \mu \mathrm{m}$ wide nozzles could reduce total encapsulation time by sixfold compared to a single-nozzle device. Microgels with sub$100 \mu \mathrm{m}$ diameters are ideal for many applications, due to their relatively low polymer content, but encapsulating clinically relevant numbers of cells in small microgels poses unique challenges. The scheme presented here addresses these challenges in a practical but novel way, through simultaneously maximizing cell concentration and effectively parallelizing encapsulation on flow focusing nozzles.

\section{MATERIALS AND METHODS}

\section{Microfluidic fabrication}

Photolithography was used to pattern microfluidic masters after spin coating SU-8 2050 photoresist (MicroChem, Westborough, MA, USA) onto silicon wafers, then masters were developed and treated with vapor phase trichloro $(1 \mathrm{H}, 1 \mathrm{H}, 2 \mathrm{H}, 2 \mathrm{H}$-perfluorooctyl) silane (Sigma, St. Louis, MO, USA) to reduce sticking. Devices with 35 and $100 \mu \mathrm{m}$ wide nozzles had focusing layer patterns that were 35 and $100 \mu \mathrm{m}$ tall, respectively, and their corresponding top layer patterns were 80 and $140 \mu \mathrm{m}$ tall. PDMS (Sylgard 184) was mixed according to manufacturer specifications and was molded on the SU-8 master to make microfluidic channel replicas (Figure 1a). After curing for $11 \mathrm{~min}$ at $110^{\circ} \mathrm{C}$, crosslinker inlet and outlet holes were punched in the top layer, and inter-layer vias were punched in the bottom layer. Relevant surfaces were exposed to air plasma for $20 \mathrm{~s}$ in a PlasmaPreen (North Brunswick, NJ, USA) system, and layers were aligned and pressed together by hand before being cured at $110^{\circ} \mathrm{C}$ for $5 \mathrm{~min}$. After PDMS to PDMS bond was stabilized, oil and macromer inlets were punched through both layers. These elastomer constructs were sonicated, along with glass substrates, in 200 proof ethanol, and then were dried with compressed nitrogen. Cleaned components were quickly treated with $12 \mathrm{~s}$ of plasma and PDMS was bonded to a glass slide, sealing the lower layer flow channels. Devices were cured overnight at $60{ }^{\circ} \mathrm{C}$ before being used.

\section{Microgel synthesis and characterization}

Microfluidic devices were primed with light mineral oil (Sigma) containing 2\% SPAN80 (Sigma), which was also used as the continuous phase fluid. The crosslinker emulsion was comprised of $30 \mathrm{mg} \mathrm{mL}^{-1}$ dithiothreitol (DTT; Sigma) emulsified at a 1:15 ratio in the continuous phase. The macromer solution consisted of 6.5\% (w/v) PEG-4MAL (20 kDa, Laysan Bio, Arab, AL, USA), which had been reacted with a cell adhesive peptide $(1.0 \mathrm{mM}$, Genscript, Piscataway, NJ, USA) that was pre-conjugated to AlexaFluor594 (Thermo Fisher, Waltham, MA, USA) to enable imaging and for consistency with cell encapsulation experiments. Also motivated by consistency with subsequent cell encapsulation experiments, this reaction was performed in a buffer which had additives to address challenges associated with cell encapsulation. Particularly, the buffer contained OptiPrep density gradient (Sigma) and PBS in a 1:4 ratio to match the density of cells and minimize settling of cells. The buffer also contained $0.1 \%$ Pluronic F108, which reduces cell sticking to glass or PDMS. After connecting the outlet and all inlets, fluid flows were driven at the flow rates prescribed in Table 1 by Hamilton gastight syringes on Harvard Apparatus Elite syringe pumps. To prevent unwanted gelation of polymer precursor on chip and minimize polydispersity due to transient 


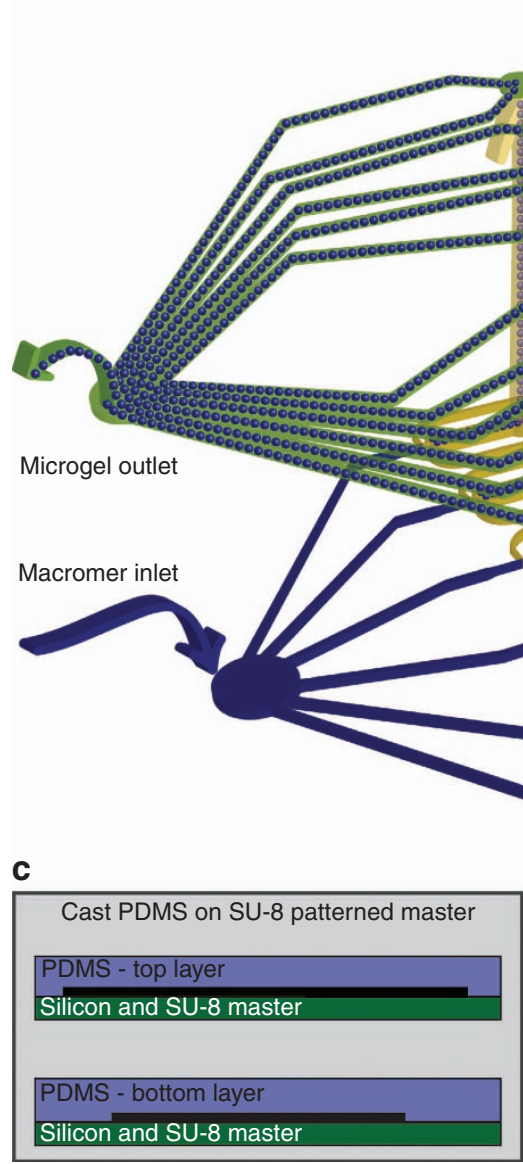

b

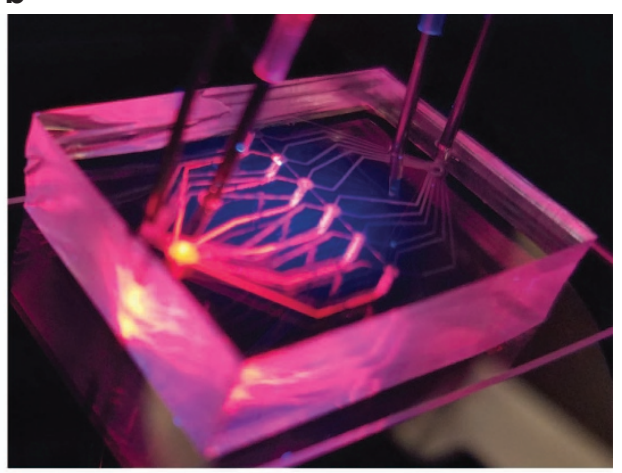

Figure 1 Schematic and representative images demonstrating parallel microfluidic device operation and fabrication are shown. (a) Droplets containing macromer precursor (and optionally cells) are emulsified in oil on the lower layer of the device when these immiscible fluids are coflowed through a flow focusing geometry. The droplets produced on all six flow focusing nozzles are carried up to the top layer where they are exposed to crosslinker and are pooled before exiting the device at a single outlet. (b) Fluorescent label facilitates visualization of microfluidic device operation, as seen in this photograph of microgel generation in progress. (c) The two-layer microfluidic device requires several steps for fabrication. First, PDMS is molded from SU-8 and silicon masters, and then holes that go through one layer only are punched. PDMS layers are exposed to air plasma and are aligned and bonded to each other. Inlets for the bottom layer are punched through both layers, and the device is completed by bonding the two-layer PDMS construct to a glass substrate after plasma exposure.

Table 1 Microfluidic devices with parallel nozzle configuration have $600 \%$ increased macromer throughput rate over single-nozzle configurations

\begin{tabular}{lccc}
\hline & \multicolumn{3}{c}{ Flow rate $\left(\mu \mathrm{L} \mathrm{min}^{-1}\right)$} \\
\cline { 2 - 4 } & Macromer & Oil & Crosslinker \\
\hline $35 \mu$ m width & & & \\
$\quad$ Single nozzle & 0.2 & 1.0 & 2.0 \\
Parallel nozzles & 1.2 & 6.0 & 15.0 \\
$100 \mu$ m width & & & \\
$\quad$ Single nozzle & 1.67 & 12.5 & 15.0 \\
Parallel nozzles & 10.0 & 75.0 & 40.0 \\
\hline
\end{tabular}

Flow rates shown were used for all experiments discussed herein.

flows, first oil, then crosslinker fluid flows were initiated and allowed to stabilize. Only after flows stabilized was macromer precursor flow initiated. After at least 20 min of generation, microgels were collected in phosphate buffered saline containing $2 \%$ bovine serum albumin (to reduce microgel sticking). Most of the oil was removed by aspiration after centrifugation (600 $\mathrm{g}$ for $5 \mathrm{~min}$ ), after which microgels were washed three more times by centrifugation in a new $15 \mathrm{~mL}$ conical tube to remove any mineral oil residue from microgels. Microgels were imaged using a Nikon Ti-E microscope with C2-Plus Confocal System, and diameter was determined using 'Analyze Particles' on binarized images in ImageJ. Output of three independent microgel generations were characterized for all four device designs tested, with a minimum of 163 microgels counted per device.

\section{Human MSC microencapsulation and characterization}

A similar protocol was used to encapsulate hMSCs, with a few modifications. First, hMSCs were trypsinized for $10 \mathrm{~min}$ in $0.25 \%$ trypsin/EDTA, which minimized cell clumping and homogenized cell morphology compared to shorter trypsinization times. A macromer solution was made containing twice the desired final concentrations-that is, $13 \mathrm{wt} \%$ PEG-4MAL, $2.0 \mathrm{mM}$ RGD, $0.2 \%$ Pluronic F108, in a buffer comprised of 1:2 OptiPrep in PBS. This precursor was mixed with an equal volume of cells suspended at twice their desired concentration. The resulting solution contained gel precursor concentrations consistent with those used for microgel synthesis and contained the desired concentration of cells in suspension $\left(2 \times 10^{7}\right.$ cells per $\mathrm{mL}$ for $35 \mu \mathrm{m}$ wide nozzle or $10^{7}$ cells per $\mathrm{mL}$ for $100 \mu \mathrm{m}$ wide nozzle). This suspension was mixed thoroughly by pipetting and was used as quickly as 
possible to generate microgels in parallel nozzle microfluidic devices, using the same protocol described for empty microgels. Three independent encapsulations were performed on both the 35 and $100 \mu \mathrm{m}$ wide nozzles for at least $20 \mathrm{~min}$ each, and the resulting microgels were washed with culture media four times by centrifugation. Live and dead cells were then stained with CalceinAM and TOTO-3 iodide, respectively (Thermo Fisher). Microgels containing cells were imaged on a confocal microscope and were measured in ImageJ. The number of cells per microgel was determined by manual counting due to cell clustering. At least 425 microgels were characterized from each encapsulation.

\section{Human MSC viability in culture}

Viability of microencapsulated hMSC was monitored over time for cells encapsulated on parallel microfluidic devices with both 35 and $100 \mu \mathrm{m}$ wide nozzles. Cell viability on day 0 was calculated by dividing the number of Calcein-AM positive cells by the total number of cells counted. Some encapsulated cells were not stained on day 0 and were instead cultured with media changes every three days. On days 1, 3 and 7 post encapsulation, encapsulated cells were removed from culture and were stained and imaged to determine cell viability. As a control, viability was also determined over time for hMSCs grown on TC plastic. Day 0 viability of cells in microgels was compared for cells encapsulated on $35 \mu \mathrm{m}$ vs $100 \mu \mathrm{m}$ wide nozzle devices using Mann-Whitney test in GraphPad (La Jolla, CA, USA).

\section{VEGF secretion}

Twenty four hours following encapsulation, $\sim 10^{5}$ hMSCs, microencapsulated using either $35 \mu \mathrm{m}$ or $100 \mu \mathrm{m}$ nozzle devices, were suspended in $1.0 \mathrm{~mL}$ growth media in a 24-well plate. Unencapsulated hMSCs were also seeded onto a 24-well plate at an equal concentration. After incubation for $48 \mathrm{~h}$, conditioned media from all samples was collected and frozen at $-80^{\circ} \mathrm{C}$. At the time of analysis, conditioned media was thawed and concentrations of VEGF were quantified using ELISA (Biolegend, San Diego, CA, USA). DNA was quantified from each sample using CyQUANT assay following the manufacturer's instructions (Thermo Fisher). VEGF secretion was normalized to DNA content, and normalized values were compared between groups using ANOVA with a Tukey post hoc test. $P<0.05$ was considered significant.

\section{RESULTS AND DISCUSSION}

\section{Microfluidic design and fabrication}

Our target application during device development was intravascular cell delivery for extended local VEGF secretion and mitigation of inflammation. Therefore, the goals of our iterative design were to maximize encapsulation throughput as well as viability and function of encapsulated cells while simultaneously minimizing microgel size as well as the number of empty microgels. During the course of preliminary experiments with devices comprised of single square flow focusing nozzles, nozzle widths smaller than $30 \mu \mathrm{m}$ did not consistently encapsulate cells, especially hMSCs, which were relatively large cells with heterogeneous morphology. Therefore, $35 \mu \mathrm{m}$ wide nozzles were determined to be the smallest nozzle appropriate for encapsulation of hMSCs. Also during these preliminary experiments, we observed that cell viability was reduced in these very small microgels, which might limit their potency. Cells encapsulated on $100 \mu \mathrm{m}$ wide nozzles had higher viability, and therefore therapeutic potential, but encapsulation of clinically relevant cell numbers was still challenging. For example, encapsulation of 30 million cells would require $\sim 30 \mathrm{~h}$.

Therefore, we determined that parallel flow focusing geometries and very high cell suspension concentrations were essential for encapsulating clinically relevant cell numbers in a practical time frame. Several different two-layer parallel nozzle flow focusing microfluidic devices were designed and fabricated (for both 35 and $100 \mu \mathrm{m}$ widths) using soft lithography, in an iterative design process. However, at high cell loading $\left(>5 \times 10^{6}\right.$ per $\left.\mathrm{mL}\right)$, all devices tested which had more than six channels produced extremely heterogeneous microgel sizes due to increased clogging of nozzles with cells. On the six-nozzle device, clogs with deformable particles frequently cleared themselves due to increasing local pressure; however, on devices with more than six nozzles, sustained clogs were frequently observed, likely because there were channels with lower resistance through which flow could be diverted. These nozzle obstructions would cause oil to divert upstream into the aqueous phase distribution, which perturbed droplet production at multiple nozzles. On the final design (Figure 1), all flow channels on the top layer are relatively large and open $(80 \mu \mathrm{m}$ tall for devices with $35 \mu \mathrm{m}$ wide nozzles; $140 \mu \mathrm{m}$ tall for devices with $100 \mu \mathrm{m}$ wide nozzles), to reduce the pressure stressing the PDMS to PDMS bond. The six nozzle designs represent a compromise achieved through extensive trial and error to balance (a) high cell loading, (b) low empty microgel rate and (c) high cell throughput.

Maximum flow rates of macromer phase resulting in consistent microencapsulation and low polydispersity were determined in previous experiments utilizing mineral oil as the continuous phase. Fluorinated oils produce lower capillary number flow relative to mineral oil, and so can be operated with higher macromer phase flow rates while maintaining dripping flow. We attempted to increase throughput even further by utilizing one such oil, Fluorinert FC40, along with a nonionic fluorinated surfactant purchased from RAN biotechnologies (008) (Beverly, MA, USA). Unfortunately, nozzle-to-nozzle variability in droplet breakup was much greater with fluorinated oils than with mineral oil when utilizing parallel flow focusing nozzle geometries, resulting in more polydispersed droplet diameters. Because of this increased variability and difficulty of maintaining crosslinker emulsion in fluorinated oils, mineral oil was used as the continuous phase for all subsequent experiments.

\section{Parallel encapsulation allows for $600 \%$ increased throughput while maintaining size control of microgels}

A major benefit of synthesizing microgels with droplet microfluidics is the ability to precisely control microgel size and polydispersity. However, maintaining low polydispersity is challenging in parallel nozzle devices, because any variation in fluidic resistance between flow focusers (or respective distribution/ collection channels) results in variation of droplet size produced at each nozzle. We synthesized PEG-4MAL microgels on both single- and parallel nozzle devices in order to characterize any increase in polydispersity due to parallel synthesis (Figure 2). Parallel nozzle devices were operated with six times the flow rate of single-nozzle devices (Table 1). Three separate synthesis runs were performed on three separate devices of each design. Representative histograms of microgel size are shown for each synthesis run (Figures $2 \mathrm{a}$ and b) along with representative fluorescent images of microgels. Although Kruskal-Wallis tests indicate differences in median microgel size across devices of the same design ( $P<0.05$ for all four devices), this is likely due to the small variances and large sample sizes. No gross differences in size distribution were seen between microgels generated on multiple devices of the same design (Figure 2), and mean microgel size was highly consistent between devices of the same design (Table 2). For practical purposes, we conclude that microgel production is repeatable on all four microfluidic designs. Microgels analyzed from all three encapsulations were pooled for each device so that comparisons could be made between single and parallel devices with the same nozzle size. For the $35 \mu \mathrm{m}$ wide nozzle 
device, a $t$-test revealed statistical differences ( $P=0.012$; Figure $2 \mathrm{c}$ ) in mean diameter of microgels produced on parallel vs. single nozzles, but the magnitude of this difference was small $(<1.8 \%)$ -average diameter was $65.1 \pm 8.2 \mu \mathrm{m}$ for parallel nozzle devices vs. $64.0 \pm 6.3 \mu \mathrm{m}$ for single-nozzle devices (Table 2). The mean diameter was equivalent for pooled microgels produced on

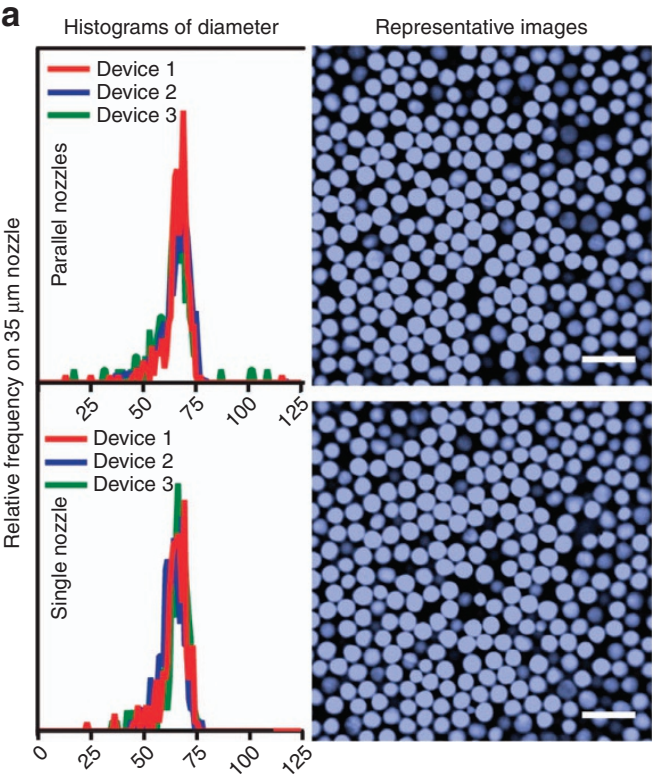

Microgel diameter $(\mu \mathrm{m})$

b
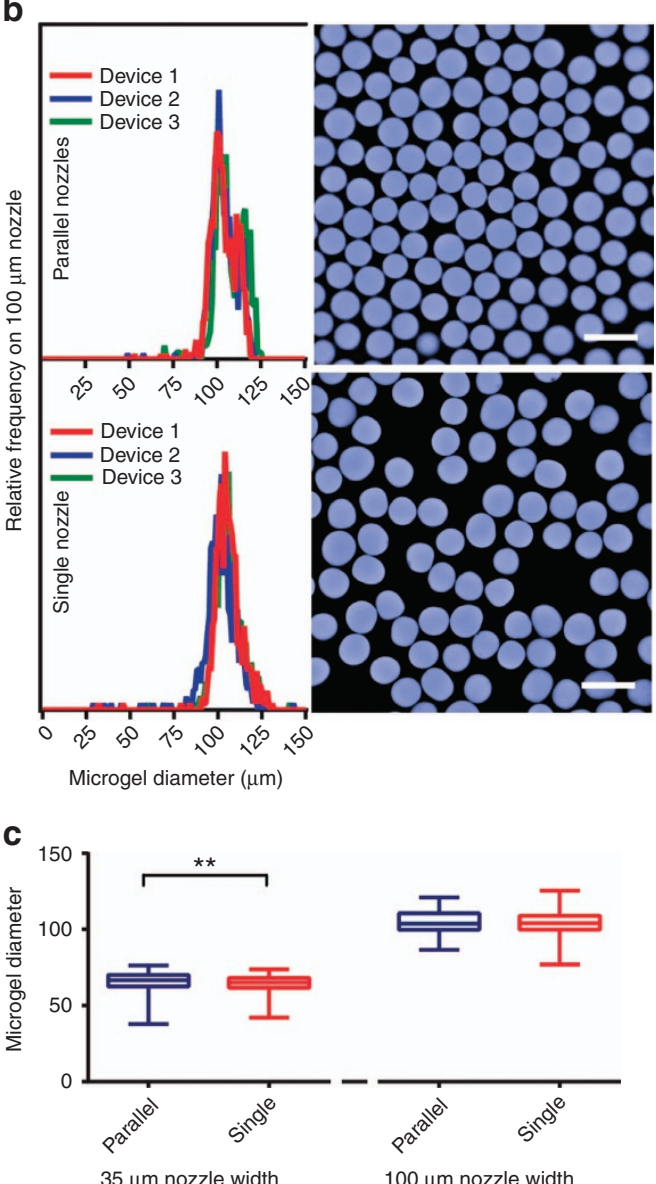

$100 \mu \mathrm{m}$ nozzles $(104.5 \mu \mathrm{m})$, whether single or parallel configurations were used, and no differences in mean diameter were found $(P=0.97)$. F-tests revealed differences in variance of microgel diameter for parallel vs. single-nozzle production for both nozzle widths tested ( $P<0.0001$ in each case). For $35 \mu \mathrm{m}$ nozzle width, the parallel configuration resulted in higher standard deviations than the single configuration, as expected. Surprisingly, the single $100 \mu \mathrm{m}$ nozzle produced microgel diameters with higher standard deviations $( \pm 9.6 \mu \mathrm{m})$ than those produced on the parallel configuration $( \pm 7.5 \mu \mathrm{m})$. Whereas statistically significant differences were found between diameters of microgels produced on single vs. parallel nozzle configurations, the differences are small and are unlikely to matter for practical applications. Taken together, these results demonstrate that our parallel microfluidic device is capable of increasing microgel production rate by $600 \%$, while having minimal impact on the size or polydispersity of resulting microgels.

Table 2 Statistics for microgel populations produced on different microfluidic devices, with or without cells

\begin{tabular}{|c|c|c|c|c|}
\hline & Device 1 & Device 2 & Device 3 & $\begin{array}{l}\text { Pooled } \\
\text { microgels }\end{array}$ \\
\hline \multicolumn{5}{|l|}{$35 \mu \mathrm{m}$ width } \\
\hline \multicolumn{5}{|l|}{ Single nozzle (-cell) } \\
\hline Mean diameter $(\mu \mathrm{m})$ & 64.2 & 62.9 & 64.7 & 64.0 \\
\hline \pm s.d. $(\mu \mathrm{m})$ & 6.0 & 5.3 & 6.8 & 6.3 \\
\hline$\overline{C V}(\%)$ & 9.3 & 8.4 & 10.6 & 9.9 \\
\hline \multicolumn{5}{|l|}{ Parallel nozzles (-cell) } \\
\hline Mean diameter $(\mu \mathrm{m})$ & 65.6 & 65.1 & 64.2 & 65.1 \\
\hline \pm s.d. $(\mu \mathrm{m})$ & 7.9 & 8.5 & 8.8 & 8.2 \\
\hline CV (\%) & 12.1 & 13.0 & 13.7 & 12.7 \\
\hline \multicolumn{5}{|l|}{ Parallel nozzles (+cell) } \\
\hline Mean diameter $(\mu \mathrm{m})$ & 77.2 & 76.4 & 75.9 & 76.5 \\
\hline \pm s.d. $(\mu \mathrm{m})$ & 6.3 & 5.7 & 7.5 & 6.6 \\
\hline$\overline{C V}(\%)$ & 8.2 & 7.5 & 9.9 & 8.6 \\
\hline \multicolumn{5}{|l|}{$100 \mu \mathrm{m}$ width } \\
\hline \multicolumn{5}{|l|}{ Single nozzle (-cell) } \\
\hline Mean diameter $(\mu \mathrm{m})$ & 106.0 & 102.0 & 105.5 & 104.5 \\
\hline \pm s.d. $(\mu \mathrm{m})$ & 12.2 & 12.7 & 8.4 & 10.1 \\
\hline $\mathrm{CV}(\%)$ & 11.5 & 12.5 & 7.9 & 9.6 \\
\hline \multicolumn{5}{|l|}{ Parallel nozzles (-cell) } \\
\hline Mean diameter $(\mu \mathrm{m})$ & 103.6 & 104.0 & 107.1 & 104.5 \\
\hline \pm s.d. $(\mu \mathrm{m})$ & 7.0 & 6.6 & 7.3 & 7.9 \\
\hline CV (\%) & 6.7 & 6.3 & 6.8 & 7.5 \\
\hline \multicolumn{5}{|l|}{ Parallel nozzles (+cell) } \\
\hline Mean diameter $(\mu \mathrm{m})$ & 97.8 & 98.1 & 98.3 & 98.1 \\
\hline \pm s.d. $(\mu \mathrm{m})$ & 7.6 & 7.2 & 6.6 & 7.1 \\
\hline $\mathrm{CV}(\%)$ & 7.8 & 7.3 & 6.7 & 7.3 \\
\hline
\end{tabular}

Results are shown for three independent syntheses for each process. Measured values from all three syntheses are pooled in the right most column to facilitate comparison between designs.

Figure 2 Microfluidic devices with parallel nozzles can produce microgels with controlled size, and with polydispersities comparable to microgels produced on a single nozzle. (a and $\mathbf{b})$ Representative histograms of microgel diameter frequency and representative fluorescent images of microgels show similar output from single and parallel nozzles for both nozzle widths tested. (c) Consistency in diameter distribution is further demonstrated by comparing pooled microgels produced from each device. Significant differences were found between parallel and single configurations of $35 \mu \mathrm{m}$ nozzles $(P=.0012)$, but the magnitude of differences was not practically relevant. $100 \mu \mathrm{m}$ nozzles did not produce different sized microgels with parallel vs. single configurations $(P=0.97)$. 


\section{Human MSCs can be encapsulated on parallel nozzle microfluidic device with high loading density}

The design criteria of maximum cell throughput and the desire for reduced transplant graft volume and microgel size both motivate a minimization of empty microgels and a maximization of cell loading in each microgel. Increasing the cell concentration in macromer solution exacerbates several challenges that may prevent successful encapsulation. High concentrations of hMSCs tend to aggregate in solution, which often result in many empty microgels and a relatively few, large microgels that contain clusters of cells. The tendency of hMSCs to aggregate was reduced by increasing trypsin exposure from $5 \mathrm{~min}$ to $10 \mathrm{~min}$ while harvesting cells, without notable deleterious effects on cell viability. Cells, especially when aggregating, tended to stick in microfluidic channels, forming sieves that prevent successful encapsulation. The addition of $0.1 \%$ Pluronic F108 eliminated this problem, along with further reducing cell aggregation. Cells also tended to settle in microfluidic tubing because their density is higher than a 6.5\% PEG-4MAL solution in PBS, which results in increased cell aggregation and many empty microgels. After adding the cytocompatible density gradient media OptiPrep at a 1:4 ratio in cell suspensions, encapsulation runs up to 30 min were completed without noticeable cell settling. These three process improvements enabled microencapsulation of hMSCs with relatively high loading densities, as shown by fluorescent images in Figure 3a. In these images, green indicates live cells (stained with Calcein-AM), red indicates dead cells (stained with TOTO-3 iodide) and blue indicates the outline of microgels (obtained using 'Find Edges' in ImageJ). Histograms showing the frequency of microgels containing a given number of cells are shown in Figure $3 \mathrm{~b}$, along with the frequencies predicted by a Poisson distribution, using the measured average value as the expected value. Measured frequencies for number of cells per microgel closely matched those predicted by a Poisson distribution for microencapsulation on both 35 and $100 \mu \mathrm{m}$ wide nozzles, except that approximately two times more empty microgels are produced than expected on $35 \mu \mathrm{m}$ wide nozzles. Fewer empty microgels are produced when $100 \mu \mathrm{m}$ wide nozzles are used rather than $35 \mu \mathrm{m}$ wide nozzles $(P=0.02$, Figure $3 c)$, which may lead to more uniform cell spacing in packed microgel arrays.

The diameters of microgels that encapsulate hMSCs were measured from three separate parallel microfluidic devices for both 35 and $100 \mu \mathrm{m}$ nozzle widths (Table 2). Cell-laden microgels produced on $100 \mu \mathrm{m}$ wide nozzles had significantly smaller diameter than cell-free microgels, with a modest decrease in mean diameter of $6.4 \mu \mathrm{m}$, or $6.1 \%$. Microgels produced on $35 \mu \mathrm{m}$ wide nozzles were significantly larger when they contained cells than when no cells were present $(P<0.0001)$, with an increase in mean diameter of $11.4 \mu \mathrm{m}$, or $18 \%$. Whereas aggregation of cells could explain the larger size of microgels produced on $35 \mu \mathrm{m}$ wide nozzles, increased polydispersity would be expected if this was the case. Surprisingly, the addition of cells decreased microgel polydispersity when compared to microgels with no cells, as seen by the lower coefficient of variation on $35 \mu \mathrm{m}$ wide nozzles when cells were present. The presence of cells did not meaningfully impact polydispersity of microgels from $100 \mu \mathrm{m}$ wide nozzles, although the $F$-test shows statistically significant differences in variance between cell-laden and cell-free microgels $(P<0.0001$ for both nozzle sizes; data in Table 2).

\section{Microgels support hMSC viability and VEGF secretion, but the microencapsulation process reduces cell viability in a nozzle size-dependent fashion}

Human MSCs were encapsulated in microgels utilizing three separate devices in independent encapsulations, for both 35 and $100 \mu \mathrm{m}$ nozzle widths with parallel configurations. These microgels were functionalized with a peptide that contained the cell adhesive sequence RGD to support adhesive signaling of encapsulated cells. On days 0, 1, 3 and 7 after encapsulation, live and dead cells were labeled with Calcein-AM and TOTO-3 iodide, respectively, before being imaged, and viable cell fractions were calculated as the fraction of live cells over the total number of cells counted for each sample (Figure 4a). Cells encapsulated on $100 \mu \mathrm{m}$ wide nozzles had excellent initial viability of $<90 \%$ on the day of encapsulation, and viability remained high ( $<75 \%)$ on day 3 post encapsulation, indicating that microgels support cell health. In contrast, cells encapsulated on $35 \mu \mathrm{m}$ wide nozzles average only $71 \%$ viability on the day of encapsulation, implicating the smaller dimensions of the device as damaging to cells. This damage could be due to increased probability of cells being exposed to membrane-disrupting mineral oil during encapsulation, which has been shown to decrease encapsulated cell viability ${ }^{39}$.

To further elucidate the effects of encapsulation on hMSCs, secretion of VEGF was measured $48 \mathrm{~h}$ after encapsulation, and was compared with secretion from hMSCs on TC plastic. Secretion of VEGF, which is known to promote angiogenesis ${ }^{40}$, was reduced in a nozzle size-dependent manner consistent with viability after encapsulation (Figure $4 b$ ). This result suggests that the ability of encapsulated cells to secrete VEGF is not negatively impacted, since reduction of VEGF secretion is explained by reduction in viability.

\section{Parallel nozzle microfluidic devices enable encapsulation of clinically relevant numbers of hMSC within practical time constraints}

Maintaining cell viability during long encapsulation or sorting processes is challenging, so processing time needs to be minimized. Clinical human and preclinical large animal studies indicate that tens of million cells will likely be required for efficacy of some cell therapies ${ }^{28,29}$. The parallelized device discussed herein increases throughput by $600 \%$ without reductions in cell viability or VEGF secretion. This reduction overcomes a practical limitation of synthetic microgel encapsulation by allowing clinically relevant cell doses to be encapsulated within a standard workday.

\section{CONCLUSIONS}

Synthetic hydrogels used to deliver cells in vivo can be engineered to provide important biochemical signals to encapsulated cells and host tissue, and tuneable pore size allows for immunoisolation of encapsulated cells from host tissue. Bulk hydrogel constructs limit mass transport around encapsulated cells and are often prohibitively large when many cells are required. Microencapsulation, or the encapsulation of cells in microgels, can reduce mass transport limitations and large graft sizes associated with bulk encapsulation of cells and enables injectable delivery of cells within controlled microenvironments. All of these benefits are enhanced as microgel diameter is decreased, but volumetric throughput decreases with the cube of nozzle width, so it can be difficult to encapsulate clinically relevant numbers of cells in very small microgels. The parallel microfluidic device presented here increases microgel throughput six-fold with minimal change in polydispersity while maintaining high cell viability and secretory functions. Microencapsulation on $35 \mu \mathrm{m}$ wide nozzle devices can be done at very high cell loading $\left(2 \times 10^{7}\right.$ cells per $\left.\mathrm{mL}\right)$, which results in relatively small microgels $(76.5 \pm 6.6 \mu \mathrm{m})$, minimizing graft volume and transport limitations. The lower cell densities required to maintain uniform droplet breakup on $100 \mu \mathrm{m}$ wide nozzles would result in increased graft volumes, but associated increases in cell viability and throughput may outweigh these concerns. Secretion of VEGF is reduced by hMSC encapsulation proportionally to the nozzle size-dependent loss of viability, 
a
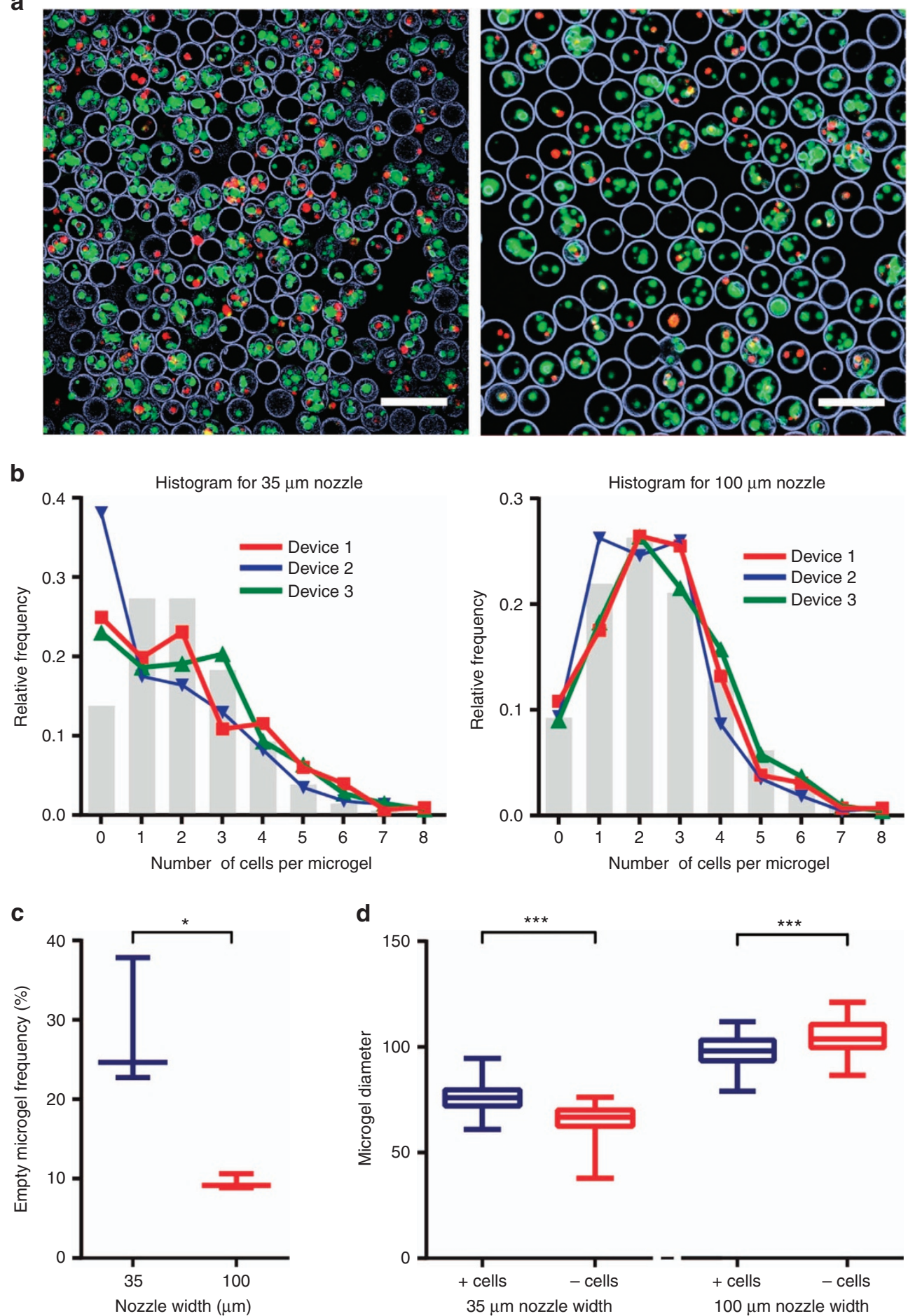

Figure 3 Parallel nozzle microfluidic devices provide high-throughput encapsulation of human mesenchymal stem cells (hMSCs) with high loading density. (a) Representative images are shown for cells microencapsulated on both $35 \mu \mathrm{m}$ (left) and $100 \mu \mathrm{m}$ (right) nozzle widths (scale bar $200 \mu \mathrm{m}$ ). Microgels encapsulating hMSCs were functionalized with a fluorescently labeled peptide containing the cell adhesive sequence RGD in order to support cell health and aid microgel visualization (outline of microgels was traced in blue using ImageJ). Live cells were stained with Calcein-AM (green) and dead cells with TOTO-3 iodide (red). (b) The number of cells per microgel was recorded for three independent encapsulations for each size nozzle, and their relative frequencies were plotted on histograms, along with the frequencies predicted by a Poisson distribution (gray bars). Number of cells in microgels produced on $100 \mu \mathrm{m}$ wide nozzles closely matched the frequencies predicted by a Poisson distribution, but microgels produced on $35 \mu \mathrm{m}$ wide nozzle devices contained more empty microgels than predicted. (c) Microencapsulation on $100 \mu \mathrm{m}$ wide nozzles produces significantly fewer empty microgels than devices with $35 \mu \mathrm{m}$ wide nozzles $(P=0.02)$. (d) Addition of cells has significant effects on distributions of microgel diameter vs. cell-free microgels for both $35 \mu \mathrm{m}$ width, where average microgel size increases, and $100 \mu \mathrm{m}$ width, where average microgel size decreases $(P<0.0001$ for both widths). 


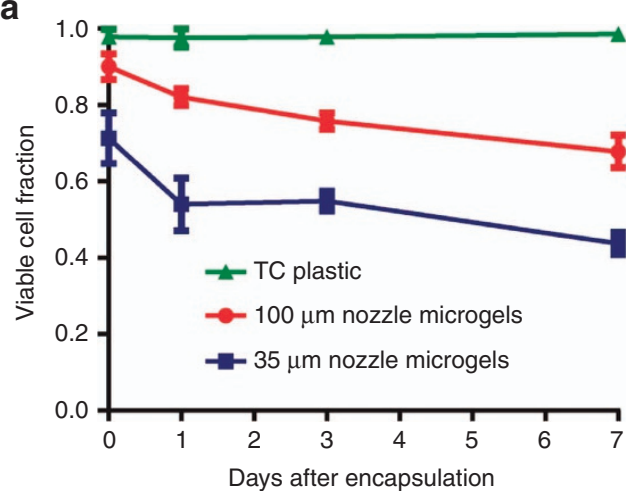

b

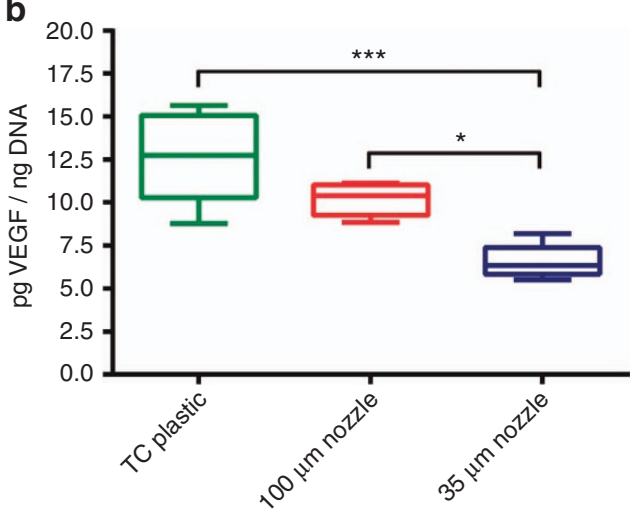

Figure 4 Microencapsulation in PEG-4MAL microgels has nozzle size-dependent effects on hMSC viability and VEGF secretion. (a) Viable cell fraction was determined over time in culture to measure the ability of microgels produced on each nozzle width to support hMSC health. Cells encapsulated on $100 \mu \mathrm{m}$ wide nozzles had good viability on the day of encapsulation, and maintained adequate viability for seven days in culture. Cells encapsulated on $35 \mu \mathrm{m}$ wide nozzles had significantly reduced viability on the day of encapsulation versus cells encapsulated on $100 \mu \mathrm{m}$ wide nozzles $(P=0.01)$, after which cell viability decreased at similar rates to cells encapsulated on $100 \mu \mathrm{m}$ wide nozzles. (b) VEGF secretion from encapsulated hMSCs followed similar nozzle sizedependent trends to viability, suggesting that VEGF secretion per viable cell remains unchanged following encapsulation. $\left({ }^{*} P<0.05\right.$; $\left.{ }^{* * *} P<0.001 ; * * * * P<0.0001\right)$. hMSCs, human mesenchymal stem cells' (hMSCs); TC, tissue culture; VEGF, vascular endothelial growth factor.

which indicates that viable cells maintain capacity to promote angiogenesis. Each nozzle width has been designed prioritizing differently the particular challenges associated with highthroughput cell encapsulation in small microgels, but both of these devices utilize similar, novel approaches to solve limited cell throughput. By using high concentration cell suspensions and parallel flow focusing nozzles, clinically relevant cell numbers can be rapidly encapsulated in small microgels.

\section{ACKNOWLEDGEMENTS}

This work was funded by NIH (Grant No. R21EB020107) and the Juvenile Diabetes Research Foundation (Grant No. 2-SRA-2014-287-Q-R).

\section{COMPETING INTERESTS}

DMH and AJG are inventors in a patent associated with polyethylene microgels for cell encapsulation (U.S. Patent 9,381,217). The remaining authors declare no conflict of interest.

\section{REFERENCES}

1 Liu AL, Garcia AJ. Methods for generating hydrogel particles for protein delivery. Annals of Biomedical Engineering 2016; 44: 1946-1958.

2 Foster GA, Headen DM, González-García C et al. Protease-degradable microgels for protein delivery for vascularization. Biomaterials 2017; 113: 170-175.

3 Lutolf MP, Hubbell JA. Synthetic biomaterials as instructive extracellular microenvironments for morphogenesis in tissue engineering. Nature Biotechnology 2005; 23: 47-55.

4 Alge DL, Azagarsamy MA, Donohue DF et al. Synthetically tractable click hydrogels for three-dimensional cell culture formed using tetrazine-norbornene chemistry. Biomacromolecules 2013; 14: 949-953.

5 Vegas AJ, Veiseh $\mathrm{O}$, Gurtler $\mathrm{M}$ et al. Long-term glycemic control using polymerencapsulated human stem cell-derived beta cells in immune-competent mice. Nature Medicine 2016; 22: 306-311.

6 Tomei AA, Manzoli V, Fraker CA et al. Device design and materials optimization of conformal coating for islets of Langerhans. Procedings of the National Academies of Sciences USA 2014; 111: 10514-10519.

7 Mao AS, Shin J-W, Utech S et al. Deterministic encapsulation of single cells in thin tunable microgels for niche modelling and therapeutic delivery. Nature Materials 2016; 16: 236-243.

8 Park JS, Chu JS, Tsou AD et al. The effect of matrix stiffness on the differentiation of mesenchymal stem cells in response to TGF-beta. Biomaterials 2011; 32: 3921-3930.

9 García JR, Clark AY, García AJ. Integrin-specific hydrogels functionalized with VEGF for vascularization and bone regeneration of critical-size bone defects. Journal of Biomedical Materials Research Part A 2016; 104: 889-900.

10 Johnson AS, O'Sullivan E, D'Aoust LN et al. Quantitative assessment of islets of langerhans encapsulated in alginate. Tissue Engineering Part C: Methods 2011; 17: 435-449.

11 Lim F, Sun AM. Microencapsulated islets as bioartificial endocrine pancreas. Science 1980; 210: 908-910.

12 Headen DM, Aubry G, Lu H et al. Microfluidic-based generation of size-controlled, biofunctionalized synthetic polymer microgels for cell encapsulation. Advanced Materials 2014; 26: 3003-3008.

13 Williams CG, Malik AN, Kim TK et al. Variable cytocompatibility of six cell lines with photoinitiators used for polymerizing hydrogels and cell encapsulation. Biomaterials 2005; 26: 1211-1218.

14 Allazetta S, Hausherr TC, Lutolf MP. Microfluidic synthesis of cell-type-specific artificial extracellular matrix hydrogels. Biomacromolecules 2013; 14: 1122-1131.

15 Kesselman LRB, Shinwary S, Selvaganapathy PR et al. Synthesis of monodisperse, covalently cross-linked, degradable 'smart' microgels using microfluidics. Small 2012; 8: 1092-1098.

16 Griffin DR, Weaver WM, Scumpia PO et al. Accelerated wound healing by injectable microporous gel scaffolds assembled from annealed building blocks. Nature Materials 2015; 14: 737-744.

17 Allazetta S, Kolb L, Zerbib $S$ et al. Cell-instructive microgels with tailor-made physicochemical properties. Small 2015; 11: 5647-5656.

18 Siltanen C, Yaghoobi M, Haque A et al. Microfluidic fabrication of bioactive microgels for rapid formation and enhanced differentiation of stem cell spheroids. Acta Biomaterialia 2016; 34: 125-132.

19 Velasco D, Tumarkin E, Kumacheva E. Microfluidic encapsulation of cells in polymer microgels. Small 2012; 8: 1633-1642.

20 Dolega ME, Abeille F, Picollet-D'hahan N et al. Controlled 3D culture in Matrigel microbeads to analyze clonal acinar development. Biomaterials 2015; 52: 347-357.

21 Tumarkin E, Tzadu L, Csaszar E et al. High-throughput combinatorial cell coculture using microfluidics. Integrative Biology 2011; 3: 653-662.

22 Agarwal $\mathrm{P}$, Choi JK, Huang $\mathrm{H}$ et al. A biomimetic core-shell platform for miniaturized 3D cell and tissue engineering. Particle \& Particle Systems Characterization 2015; 32: 809-816.

23 Anna SL, Bontoux N, Stone HA. Formation of dispersions using 'flow focusing' in microchannels. Applied Physics Letters 2003; 82: 364-366.

24 Lienemann PS, Rossow T, Mao AS et al. Single cell-laden protease-sensitive microniches for long-term culture in 3D. Lab on a Chip 2017; 17: 727-737.

25 Collins DJ, Neild A, deMello A et al. The Poisson distribution and beyond: Methods for microfluidic droplet production and single cell encapsulation. Lab on a Chip 2015; 15: 3439-3459.

$26 \mathrm{Kim} \mathrm{J}$, Won J, Song S. Dual-mode on-demand droplet routing in multiple microchannels using a magnetic fluid as carrier phase. Biomicrofluidics 2014; 8: 054105.

27 Agresti JJ, Antipov E, Abate AR et al. Ultrahigh-throughput screening in dropbased microfluidics for directed evolution. Proceedings of the National Academy of Sciences of United States of America 2010; 107: 4004-4009.

28 Strauer BE, Brehm M, Zeus T et al. Repair of infarcted myocardium by autologous intracoronary mononuclear bone marrow cell transplantation in humans. Circulation 2002; 106: 1913-1918. 
29 Golpanian S, Schulman IH, Ebert RF et al. Concise review: Review and perspective of cell dosage and routes of administration from preclinical and clinical studies of stem cell therapy for heart disease. Stem Cells Translational Medicine 2016; 5: 186-191.

30 Tetradis-Meris G, Rossetti D, de Torres CP et al. Novel parallel integration of microfluidic device network for emulsion formation. Industrial \& Engineering Chemistry Research 2009; 48: 8881-8889.

31 Bardin D, Kendall MR, Dayton PA et al. Parallel generation of uniform fine droplets at hundreds of kilohertz in a flow-focusing module. Biomicrofluidics 2013; 7: 13.

32 Jeong $\mathrm{H}-\mathrm{H}$, Yelleswarapu VR, Yadavali $\mathrm{S}$ et al. Kilo-scale droplet generation in three-dimensional monolithic elastomer device (3D MED). Lab on a Chip 2015; 15: 4387-4392.

33 Mulligan MK, Rothstein JP. Scale-up and control of droplet production in coupled microfluidic flow-focusing geometries. Microfluidics and Nanofluidics 2012; 13: 65-73.

34 Muluneh M, Issadore D. Hybrid soft-lithography/laser machined microchips for the parallel generation of droplets. Lab on a Chip 2013; 13: 4750-4754.

35 Li W, Greener J, Voicu D et al. Multiple modular microfluidic (M 3) reactors for the synthesis of polymer particles. Lab on a Chip 2009; 9: 2715-2721.

$36 \mathrm{Kim} \mathrm{SH}, \mathrm{Kim}$ JW, Kim DH et al. Enhanced-throughput production of polymersomes using a parallelized capillary microfluidic device. Microfluidics and Nanofluidics 2013; 14: 509-514.

37 Romanowsky MB, Abate AR, Rotem A et al. High throughput production of single core double emulsions in a parallelized microfluidic device. Lab on a Chip 2012 12: 802-807.

38 Amstad $\mathrm{E}$, Chemama $\mathrm{M}$, Eggersdorfer $\mathrm{M}$ et al. Robust scalable high throughput production of monodisperse drops. Lab on a Chip 2016; 16: 4163-4172.

$39 \mathrm{Kim} \mathrm{S}$, Oh J, Cha C. Enhancing the biocompatibility of microfluidics-assisted fabrication of cell-laden microgels with channel geometry. Colloids and Surfaces $B$ : Biointerfaces 2016; 147: 1-8.

40 Ferrara N, Gerber HP, LeCouter J. The biology of VEGF and its receptors. Nature Medicine 2003; 9: 669-676.

(c) (i) International License. The images or other third party material in this article are included in the article's Creative Commons license, unless indicated otherwise in the credit line; if the material is not included under the Creative Commons license, users will need to obtain permission from the license holder to reproduce the material. To view a copy of this license, visit http://creativecommons.org/licenses/ by/4.0/

(c) The Author(s) 2018 